\section{Elektrik hilft gegen Rückenschmerzen}

\section{Eine Kombination aus transkranieller Gleichstromstimulation und einer peripheren Elektrostimulation lindert die Beschwerden von Patienten mit Rückenschmerzen.}

\begin{abstract}
Sezialisten für physikalische Thera$S$ pie, angeführt von Fuad Hazime von der Universität Piauí in Parnaíba, haben in einer Studie den Effekt von transkranieller Gleichstromstimulation (TGSS) und einer peripheren Elektrostimulation (PES) auf das Beschwerdebild bei chronischen Rückenschmerzen untersucht. Die theoretische Grundlage dafür lieferten ihnen vorangegangene Befunde, wonach chronisches Kreuzweh zu plastischen Veränderungen im Gehirn führt, die sich durch Neuromodulation modifizieren lassen.

92 Patienten waren beteiligt. Sie wurden nach dem Zufall in vier Gruppen aufgeteilt. Gruppe 1 erhielt zwölf Sitzungen einer anodalen TGSS, wofür am Kopf über den Punkten C3 oder C4 im 10-20-System der Elektroenzephalografie (jeweils kontralateral zur Seite des Schmerzes) die Anode und ipsilateral zum Schmerz supraorbital die Kathode befestigt wurde. 20 Minuten lang floss
\end{abstract}

dann ein Strom von $2 \mathrm{~mA}$. Gruppe 2 bekam eine PES über vier Elektroden, bilateral parallel im Lumbalsegment mit der größten Schmerzintensität, in Pulsfrequenz von $100 \mathrm{~Hz}$ und mit einer Pulsdauer von $200 \mu$ s, über 40 Minuten hinweg. Gruppe 3 wurde sowohl die TGSS wie die PES zuteil, und Gruppe 4 erhielt Scheinbehandlungen (gleiche Anwendungen, aber nur über eine Dauer von 30 Sekunden).

\section{TGSS allein bringt zu wenig}

Den Ausgangswert der Schmerzen hatten die Patienten vor Beginn der Elektrotherapie auf einer Skala von 0 bis 10 (stärkste Schmerzen) zwischen 6 und 7 verortet. Die Kombination von TGSS und PES reduzierte die Schmerzintensität um median 2,6 Punkte, die PES um 2,2 Punkte. Die Schwelle für einen klinisch bedeutsamen Effekt liegt bei einer Veränderung von zwei Punkten. Diese Schwelle konnte mit der TGSS allein ebenso wenig überwunden werden wie mit der Placebotherapie.

Ein bis zu drei Monate anhaltender Effekt war nur mit der Kombination aus TGSS und PES zu erzielen. Keine der Behandlungsformen hatte einen Einfluss auf den Grad der Behinderung oder auf die affektiven Aspekte der Schmerzbeschwerden.

Fazit der Autoren: In der Bilanz ihrer Studie schreiben Hazime und Kollegen, PES allein oder in Kombination mit TGSS sei akut und auf kurze Sicht effektiv gegen die Schmerzen von Patienten mit chronischen unspezifischen lumbalen Rückenschmerzen. Auf mittlere Sicht hätte aber nur die Kombinationsbehandlung einen klinisch relevanten Nutzen. Die beiden neuromodulatorischen Techniken wirkten womöglich synergistisch, so die Forscher, und weiter: „Die alleinige Anwendung von TGSS gegen Kreuzweh wird durch unsere Befunde nicht gestützt.“

Dr. Robert Bublak

Hazime FA et al. Treating low back pain with combined cerebral and peripheral electrical stimulation: A randomized, double-blind, factorial clinical trial. Eur J Pain 2017; online 25. April

\section{Tumorschmerz: Bei jedem Fünften neuropathisch}

\author{
Unter ambulant versorgten Krebspatienten hat fast jeder dritte chronische \\ Tumorschmerzen. Bei jedem fünften Schmerzpatienten handelt es sich um \\ neuropathische Schmerzen mit verstärkter Intensität, wie aus einer französi- \\ schen Studie hervorgeht.
}

$B_{n}^{i}$ sherige Schätzungen zur Häufigkeit neuropathischer Schmerzen bei Patienten mit chronischen Tumorschmerzen beruhen auf Studien mit stationär behandelten Patienten in speziellen Einrichtungen wie Schmerz- und Palliativkliniken. Nach Ansicht von Dr. Didier Bouhassira vom Gesundheitsinstitut INSERM in Boulogne-Billancourt in Frankreich und seinen Kollegen sind Untersuchungen ambulanter Tumorschmerzpatienten repräsentativer.

Deshalb haben sie den Anteil der Krebspatienten mit chronischen Schmerzen mit oder ohne neuropathi- sche Schmerzen ermittelt, die in einem Zeitraum von zwei Wochen (zehn Arbeitstagen) die ambulante Abteilung einer Spezialklinik aufsuchten. Als chronisch waren Schmerzen definiert, wenn sie mindestens drei Monate anhielten. Neuropathische Schmerzen wurden mithilfe des DN4-Scores (Douleur Neuropathique en 4 Questions) ermittelt. Darüber hinaus bestimmten die Ärzte prospektiv die Inzidenz chronischer Schmerzen mit oder ohne neuropathische Schmerzen.

Eingebunden waren insgesamt zwölf Einrichtungen unter 20 Krebszentren und 25 onkologischen Abteilungen in Frankreich. Insgesamt 1.805 Patienten nahmen an der Studie teil. Patienten, die zum Zeitpunkt der ersten Untersuchung keine Schmerzen hatten, wurden zur Ermittlung der Inzidenz nach drei und sechs Monaten erneut untersucht.

\section{Neuropathische Schmerzen sind besonders quälend}

Der Auswertung der Studiendaten zufolge hatten 28,2\% der Studienteilnehmer chronische Schmerzen. Bei 47,6\% der Patienten vermuteten die Ärzte den Tumor als Schmerzursache, bei $23,7 \%$ die Krebstherapie und bei $23,5 \%$ eine tumorunabhängige Ursache. Fast jeder zweite Patient (48,7\%) klagte über Schmerzen im Rücken, 45,6\% über Schmerzen in Hals und Schultern, $43,6 \%$ in den Beinen und 36,6\% in den Armen. Die Prävalenz neuropathischer Schmerzen lag - ermittelt mithilfe des 